\title{
O Espaço à Época do Queer: contaminações queer na geografia francesa ${ }^{1}$
}

\author{
Space at the Time of Queer: queer contamination in the french geography \\ El Espacio en la Época Queer: contaminaciones queer en la geografía francesa
}

\author{
Rachele Borghi \\ Université Paris IV - França \\ rachele.bor@gmail.com
}

\section{Resumo}

O espaço não é simplesmente um pano de fundo, um quadro sobre o qual as ações humanas acontecem. Ele é também um produtor de significações e um reprodutor dos mecanismos e das dinâmicas sociais. No entanto, considerar o papel do espaço como vetor e reprodutor das normas sociais relacionadas ao gênero e às sexualidades não é algo óbvio. $\mathrm{O}$ espaço público é concebido, gerado e modelado sobre a base de uma concepção dualista rígida: homem-mulher, lícito-ilícito, homossexual-heterossexual. Dessa maneira, a natureza de gênero do espaço social é ocultada pela naturalização da divisão entre espaço público e espaço privado, reflexo da divisão da vida social entre espera pública e esfera privada. Neste artigo, proponho alguns elementos de reflexão sobre a relação entre espaço, gênero e sexualidade na geografia. Partindo de uma revisão da literatura anglófona sobre a influência das teorias queer na produção do pensamento geográfico, mostrarei suas derivações francesas, para então fazer uso do conceito de performance.

Palavras-Chave:Espaço; Queer; Geografia Francesa.

\begin{abstract}
Space is not just a backdrop, a framework on which human actions take place. It is also a producer of meanings and a reproducer of social mechanisms and dynamics. However, considering the role of space as a vector and reproducer of social norms related to gender and sexuality is not something obvious. Public space is designed, generated and modeled on the basis of a rigid dualistic conception: men and women, legal-illegal, gay-heterossexual. Thus, the gendered nature of social space is hidden by the naturalization of the division between public space and private space, reflecting the expected division of society between public and private sphere. This paper offers some thoughts on the relations between space, gender and sexuality in geography. From a review of Anglophone literature on the influence of queer theories on the production of geographical thought, I will treat on their French derivations, then make use of the concept of performance.
\end{abstract}

Keywords: Space; Queer; French Geography. 


\section{Resumen}

El espacio no es sólo un telón de fondo, un marco en el que ocurren las acciones humanas. Es también un productor de significados y un reproductor de mecanismos y dinámicas sociales Sin embargo, considerar el papel del espacio como un vector y como reproductor de las normas sociales relacionadas con el género y las sexualidades no es algo obvio. El espacio público es concebido, generado y modelado sobre la base de una concepción dualista rígida: hombre-mujer, lícito-ilícito, homosexual-heterosexual. Por lo tanto, la naturaleza de género del espacio social se oculta en la naturalización de la división entre el espacio público y el espacio privado, lo que refleja la división de la sociedad entre la esfera pública y la privada. Este artículo ofrece algunos elementos de reflexión sobre la relación entre el espacio, el género y la sexualidad en la geografía. A partir de una revisión de la literatura anglófona sobre la influencia de las teorías queer en la producción del pensamiento geográfico, mostraré sus derivaciones francesas para el uso del concepto de performance.

Palabras-Clave: Espacio; Queer; Geografía Francesa.

\section{Da Geografia do Gênero...}

Embora o gênero constitua uma categoria social fundamental para as dinâmicas socioespaciais, até os anos 1980 a geografia negligenciou a questão das relações sociais de sexo delegando a reflexão a outras disciplinas como a antropologia, a sociologia ou a história (BARTHE; HANCOCK, 2005). Marianne Blidon (2009a) relaciona essa falta de interesse da parte da geografia ${ }^{2}$ com a questão da legitimidade dos objetos/sujeitos de estudo:

Além do constrangimento que as questões sexuais ainda suscitam um dos fatores de explicação da falta de interesse dos geógrafos franceses por esses temas reside na hierarquia dos objetos de estudo, alguns julgados mais 'nobres' que outros (BLIDON, 2009a, p. 57).

Ela apoia sua crítica na reflexão de Gagnon (1992 apud BLIDON, 2009a, p. 57), que chama a atenção para a marginalização institucional dos pesquisadores que se ocupam de temáticas consideradas 'perturbadoras':
As pessoas engajadas nos estudos sociais ou culturais, que se interessam enquanto pesquisadores pela sexualidade, pelo uso de drogas, pela pobreza, pela etnicidade ou pelas mulheres são, frequentemente, tão marginais no seu meio profissional quanto são as pessoas que eles estudam na sociedade na qual vivem [...] Eles sabem diretamente, ou indiretamente, que a ciência foi um elemento importante de controle social assim como da construção da realidade social (GAGNON, 1992 apud BLIDON, 2009a, p. 57).

A geografia do gênero tem por objetivo principal a análise das relações entre espaço e gênero nas suas formas mais variadas, e nos papéis e funções que homens e mulheres ocupam nestas ${ }^{3}$. A reflexão sobre essas temáticas se desenvolveu de uma maneira estruturada a partir da segunda metade dos anos 1970. Ela teve início nas teorias feministas que analisaram o mundo do trabalho e suas divisões com base no gênero, focando o binômio trabalho de reprodução, atribuído às mulheres, e trabalho de 
produção, competência dos homens (BONDI; DOMOSH, 2001).

A geografia feminista se desenvolveu incluindo as contribuições teóricas do feminismo para a explicação e para a interpretação dos fatos geográficos ${ }^{4}$.

Um dos manifestos mais significativos é o livro Geography and Gender. An Introduction to feminist Geography, publicado em 1984 pelo 'Women and Geography Group' do Institute of British Geographers. Essa obra deu uma legitimidade científica à geografia feminista e consagrou-a definitivamente como um ramo da geografia.

Nós apoiamos a importância de introduzir a ideia da geografia feminista - uma geografia que leva explicitamente em conta o gênero criado socialmente no seio da estrutura da sociedade; ela expressa um engajamento para atenuar as desigualdades de gênero a curto prazo, e para sua eliminação, através da mudança em direção à igualdade real, a longo prazo (WGSG, 1984, p. $31)$.

Os/as autores/as introduzem quatro grandes temáticas para a geografia feminista (que está, então, dando seus primeiros passos): as transformações da estrutura urbana, o acesso aos serviços, a relação entre mulheres e desenvolvimento, e todas as temáticas que integram a dimensão de gênero às análises de geografia econômica.

É somente com o feminismo pósestruturalista que se começa a tratar da interpretação das diferenças sexuais e das construções de gênero por meio de uma abordagem desconstrutivista. A mudança epistemológica impulsiona a geografia feminista a se configurar como uma geografia do gênero, consagrada à análise mais estruturada das relações de gênero como construções sociais e aos seus efeitos no espaço. As diferenças de gênero são analisadas como fenômenos ligados às lógicas de poder (BONDI; DOMOSH, 2001).

A geografia do gênero estudou e criticou o mecanismo de produção do conhecimento, enfatizando como a disciplina operou por meio de uma epistemologia sexista ${ }^{5}$. Além disso, ela voltou sua atenção para um outro limite da geografia tradicional: crer que é possível observar o mundo 'do exterior', de forma 'objetiva'. Nesse contexto, o pesquisador (homem, branco, ocidental) é supostamente exterior à realidade observada, excluindo assim toda a subjetividade. $\mathrm{O}$ método de pesquisa é frequentemente quantitativo, o pesquisador fala na terceira pessoa e adota um estilo de escrita neutro, passivo e apoiado em uma terminologia técnica. Isso lhe permite tomar distância de todo o tipo de responsabilidade com relação ao seu próprio trabalho, afirmando a natureza não política da pesquisa científica (ROSE, 1993; DIXON; JONES III, 2006, ver também CHIVALLON, 2001; HANCOCK, 2004). Os estudos de gênero reagiram contra a ideia de que a cientificidade (e, por conseguinte, a legitimidade) dos conteúdos esteja subordinada a esse tipo de organização metodológica e estilística. Eles propuseram novas metodologias que enfatizaram, especialmente na pesquisa de campo, a componente subjetiva e as relações de influência mútua entre pesquisador/a e sujeito/objeto de pesquisa.

Essa abordagem insere-se na virada cultural que, embora tenha envolvido toda a comunidade científica ${ }^{6}$, é frequentemente percebida como associada às pesquisas consideradas 'feministas', centradas nas mulheres e conduzidas por mulheres. Além disso, ainda que a atenção tenha sido dirigida ao gênero como construção social e simbólica, a pesquisa, por muito tempo, voltou a sua atenção para o tema 'mulheres'. 
Enquanto gênero se conjuga tanto no masculino quanto no feminino, a conexão entre pensamento feminista e gênero como categoria de análise nem sempre conseguiu romper com o estudo unicamente do 'feminino'. No entanto, o gênero constitui uma categoria de análise de primeira importância: tanto para os homens como para as mulheres, o gênero segue os modelos normativos diferenciados, constituindo, por conseguinte, um objeto de interesse incontornável.

A geografia do gênero não se limitou à análise dos conceitos tradicionais da disciplina (região, paisagem, lugar, território, etc.) à luz da epistemologia feminista; ela revelou novos objetos de análise; entre estes se encontra o corpo $^{7}$. Ela se apresenta como uma subdisciplina da geografia que, como outras, é decididamente interdisciplinar. Desde os anos 1980, ela se distinguiu por três abordagens teóricas principais: o gênero como diferença, o gênero como relação social e o gênero como construção social (DIXON; JONES III, 2006).

\section{... à Geografia das Sexualidades}

Uma vez que os trabalhos científicos que levaram em consideração a categoria "gênero" a limitaram durante muito tempo ao binômio homem/mulher heterossexual, as pesquisas sobre as identidades sexuais e suas relações com o espaço ainda são raras atualmente $^{8}$. Enquanto o espaço é analisado sob o ângulo da interseccionalidade, ou seja, as interconexões entre as categorias de classe, gênero e 'raça', a sexualidade continua sendo negligenciada. No entanto, a sexualidade não diz respeito somente à dimensão privada; ela afeta os espaços do cotidiano (BLUNT; WILLS, 2000; JOHNSTON; LONGHURST, 2010) e representa um âmbito geopolítico significativo (BLIDON, 2009a).

$\mathrm{Na}$ realidade, a partir dos anos 1970, as perspectivas de pesquisa relativas à sexualidade surgiram nos estudos sobre o processo de construção dos espaços urbanos e contribuíram para a sua renovação. As formas espaciais criadas pelas comunidades gays e lésbicas se tornaram objetos de estudo. As gay villages e os bairros homossexuais das cidades dos Estados Unidos (São Francisco em particular) começaram a ser lidos como paisagens culturais, sociais e políticas e como o reflexo da experiência espacial de sexualidades 'outras' (CASTELLS; MURPHY, 1982; WEIGHTMAN, 1981). Os trabalhos que voltam sua atenção para as relações entre cultura, consumo e espaço urbano enfatizam o papel da comunidade gay no processo de gentrificação dos centros históricos (CASEY, 2004).

A geografia da sexualidade se distanciou, então, do trabalho de cartografia das zonas residenciais urbanas ${ }^{9}$ para estudar a questão mais complexa da relação entre espaço, identidades sexuais e poder. A vida e as práticas de gays e lésbicas no espaço urbano foram exploradas a fim de tornar visíveis as sexualidades 'dissidentes' e as formas de resistência à opressão da heteronormatividade (BLUNT; WILLS, 2000) ${ }^{10}$.

$\mathrm{O}$ termo heteronormatividade indica a interiorização da heterossexualidade como expressão "normal" das relações sexuais. Esse conceito permitiu reinterpretar os espaços para além das categorias tradicionais e questionar a sexualidade normativa, ou seja, aquilo que é considerado como "correto" e "normal" (e, assim, merece ser incluído no espaço público). Ao mesmo tempo, essa perspectiva permitiu refletir sobre as diferentes violações das regras da norma sexual e de gênero (WIEGMAN, 2006).

Essa 'virada sexual' em geografia foi possível graças à afirmação da teoria queer. Os queer studies permitiram renovar o estudo das relações entre gênero, sexualidade e espaço público. A partir dos anos 1990, esses 
estudos permitiram que se abandonassem as lógicas binárias (masculino/feminino, homo/hétero) e que se evidenciasse o gênero como paradoxo. Nessa perspectiva, o espaço urbano é lido como o produto de estruturas sociais que excluem os 'outros' atores da cidade e os corpos não normalizados, sendo estes últimos frequentemente associados aos sujeitos LGBTIQ $^{11}$.

A teoria queer questionou as etiquetas sexuais e evidenciou todas as variações criativas do desejo sexual e de seus objetos de desejo (DE LAURETIS, 2007). O termo queer diz respeito à fenomenologia do bizarro (em inglês esse termo significa excêntrico, obscuro, ambíguo, degenerado). Embora a linguagem da heterossexualidade normativa the tenha dado uma conotação negativa ('pederasta' especialmente), o termo foi reabilitado por aqueles/as que criticam a pretensa universalidade e naturalidade do paradigma heterossexual hegemônico (DIMEN; GOLDNER, 2002). Os teóricos queers declaram querer exercer uma subversão da ordem dominante, aquela que oprime as vozes dos 'outros' e as identidades não codificadas, jogando com os símbolos e os códigos da heterossexualidade.

Foi com Teresa de Lauretis e Judith Butler que o queer se difundiu no meio acadêmico e que a queer theory foi formalizada. Desconstrução das categorias de identidade, análise da constituição do corpo na fronteira entre materialidade e linguagem, crítica do paradigma normativo heterossexual e dos dispositivos de inclusão/ exclusão, crítica do poder e do biopoder são os eixos de reflexão desse pensamento.

Por meio da teoria queer, a heterossexualidade foi questionada. A heterossexualidade institucionalizada foi estudada na relação de seu papel na regulamentação da homossexualidade. De fato, a heterossexualidade normativa teve também um impacto violento sobre a própria heterossexualidade. Esse aspecto foi por muito tempo ignorado. A crítica homossexual evidenciou como a heterossexualidade normativa tem uma forte influência sobre a vida dos heterossexuais. O conceito de heterossexualidade compulsiva foi desenvolvido graças ao trabalho de Adrienne Rich em 1980.

Rich (1980) se perguntava por que a heterossexualidade é vista apenas como um fato biológico e não como uma escolha; se a heterossexualidade poderia ser considerada uma escolha ou se trataria de uma imposição social e política; e se a heterossexualidade, assim como a maternidade, seria uma instituição política demasiado estruturada.

$\mathrm{O}$ discurso heteronormativo é agressivo para com as formas de sexualidades que se afastam da heterossexualidade normativa. Ao prescrever os comportamentos a não adotar, ele codifica simultaneamente os comportamentos considerados como 'normais' e 'corretos'. Os sujeitos LGBTIQ são marginalizados por esse discurso do mesmo modo que os heterossexuais que adotam práticas vistas como desviantes da norma. Dessa maneira, as pessoas que aderem ao modelo heterossexual são obrigadas a se conformar e a assumir todas as atitudes e todos os comportamentos que caracterizam a 'feminilidade' e a 'virilidade' normativas. Dessa forma, a identidade heterossexual influencia o controle físico dos corpos simultaneamente ao controle das instituições estatais e da cultura hegemônica (MCDOWELL; SHARP, 1999). Isso se traduz por uma gestão muito normativa dos espaços. A análise geográfica dessas questões nos permite tornar visível a maneira pela qual a heteronormatividade se inscreve espacialmente, como ela se reproduz e se legitima pelas práticas e pelas performances dos indivíduos. 


\section{Sobre a Performance}

Em 1990, Judith Butler publica pela editora Routledge Gender trouble. Esse livro tornou-se referência obrigatória da teoria queer. A autora esboça aí uma teoria da performatividade de gênero por meio de uma releitura do conceito de performance, que toma emprestado da linguística $e$ da antropologia.

Victor Turner (1982), em From Ritual to Theatre, afirmou que:

O termo 'performance' vem de 'parfournir', que, no francês antigo, significa literalmente 'fornecer completamente ou exaustivamente'. 'Performar' significa, portanto, produzir, completar qualquer coisa, executar uma ordem ou concluir um projeto. Mas, do meu ponto de vista, a execução pode gerar algo novo. A performance transforma a si próprio. [...] As regras servem-lhe de moldura, mas o 'fluxo' da ação e da interação no interior dessa moldura pode levar a novas ideias e gerar novos símbolos e significantes, que podem ser incorporados em futuras performances (TURNER, 1982, p.14).

A performance tem, portanto, um caráter experimental e, ao mesmo tempo, crítico: graças à ação, é possível viver e completar uma experiência, e, pelo ângulo da encenação do nosso corpo, refletir sobre a própria experiência.

Turner (1987) afirma que a antropologia da performance é uma parte essencial da antropologia da experiência, que cada tipo de performance cultural, incluindo o ritual, a cerimônia, o carnaval, o teatro e a poesia, é a explicação da própria vida. A performance torna visível algo que, em condições normais, é selado hermeticamente, inacessível à observação e ao raciocínio cotidiano, enterrados nas profundezas da vida sociocultural.

Com Judith Butler, o conceito de performance torna-se um leitmotiv dos queer studies $^{12}$. Butler $(2006 ; 2008 ; 2009)$ se detém sobre os fundamentos teóricos da teoria queer e, por conseguinte, sobre o conceito de performatividade. O termo é utilizado para indicar $o$ poder que certas expressões linguísticas têm de fazer acontecer a ação no momento mesmo em que são enunciadas. Essa definição é atribuída às teorias de linguagem de John L. Austin (1982).

A principal novidade introduzida por Austin na história das ideias linguísticas consiste no convite a encarar a linguagem não mais, ou não somente, como um instrumento para descrever um estado (exterior ou espiritual), mas, sobretudo, como uma ação. Os atos performativos são componentes dos atos rituais aos quais são aplicáveis critérios específicos de avaliação (PASQUINO, 2011).

$\mathrm{Na}$ perspectiva de Butler, os atos performativos são formas do discurso autoritário, pois não somente executam uma ação, mas conferem um poder coercitivo à ação executada: "Se o poder do discurso de produzir aquilo que ele nomeia está ligado à performatividade, então a expressão performativa é um domínio na qual o poder age como discurso" (BUTLER, 1993, p. 2).

Para Butler, o gênero é um enunciado performativo que tem a capacidade de criar aquilo que nomeia. Concebido como uma atribuição normativa, a performance cita gestos, posturas, palavras, as reitera, as repete e termina, ao reproduzi-las sem cessar, por naturalizar o gênero. $O$ gênero é, portanto, uma performance porque não existe antes de ser atuado. Ele não é uma qualidade do sujeito, mas uma citação da norma. Por conseguinte, o que permite a existência da 
norma é o fato de a citar e de a repetir. Nesse contexto, o sexo é o antecedente artificial do gênero; ele não é 'natural', mas ao contrário 'naturalizado' e invisibilizado pelo poder do discurso. Consequentemente, o gênero é sempre uma imitação, mas uma imitação sem original: é o ato de imitação, a performance, a citação que cria a noção de um original antecedente (ARFINI, 2011).

A análise de Butler sobre a ontologia do gênero questionou a naturalidade do binarismo sexo/gênero na qual a identidade de gênero (masculino/feminino) é concebida em relação a dois sexos naturais: macho/fêmea. Essa oposição binária é a base do desejo heterossexual. Butler afirma, também, que os sexos são construções sociais e que essa divisão binária (um sexo e seu oposto), não tem nada de 'natural'. Para ela, a identidade de gênero não corresponde a uma essência biológica. Para articular a desconstrução do binômio identidade de gênero/essência biológica, ela mobiliza $o$ conceito de performance que se torna uma ferramenta crítica para desnaturalizar as categorias sociais e para desestabilizar as formas dominantes de reprodução social. Segundo sua reflexão, o 'doing', o 'fazer' do discurso, os conhecimentos pré-constituídos e as repetições produzem os sujeitos sociais. Por conseguinte, as identidades não existem antes da performance (GREGSON; ROSE, 2000).

Butler critica a posição feminista baseada na diferença e chama a atenção para a necessidade de combater não a dominação masculina, mas, sobretudo, o paradigma heterossexual. A definição de sexo no âmbito da cultura ocidental impediu a compreensão profunda das relações de poder ligadas à heterossexualidade

(PASQUINO; PLASTINA, 2009). O paradigma heterossexual transformou-se em norma. Ele não se reforça somente graças à exclusão e à sanção das 'transgressões', mas também graças à linguagem que permite enunciar a norma, repeti-la e também naturalizá-la.

\section{Geografia e Performance}

O corpo torna-se ferramenta ou, em uma escala micro, o lugar onde a performance ganha vida. É por isso que o estudo da performance é estritamente ligado ao dos corpos e a seu lugar no espaço. O corpo, como lugar ou localização da individualidade e da construção dos sujeitos, é implicado nas mesmas dinâmicas de poder que aquelas que definem a normatividade do espaço, separando os corpos que 'contam' daqueles que não possuem as características exigidas (BUTLER, 2009).

No entanto, se, por um lado, há uma dimensão limitante da linguagem ligada à performatividade, por outro, essa última dá a possibilidade de uma mudança social e política, pois podemos sempre inverter o valor da performance e utilizá-la para afirmar, mostrar, tornar visível ou simplesmente centrar a atenção sobre algo. É o caso, por exemplo, dos hate speech, dos insultos que podem ser utilizados de maneira deformada, criativa e positivamente desviante ${ }^{13}$ (BUTLER, 2004).

A relação entre performance e espaço foi explorada na geografia anglo-saxônica repetidamente. De fato, a ideia de Butler de performatividade influenciou fortemente a geografia crítica no intuito de desnaturalizar as "evidências" das práticas sociais. Performance e performatividade são ferramentas conceituais para a geografia crítica porque permitem desnaturalizar as ideias adquiridas sobre as práticas sociais (GREGSON; ROSE, 2000). Em geografia, a performance designa um corpo em ação que produz perpetuamente uma nova realidade (THRIFT, 2000): 
A performance se refere à prática concreta de um indivíduo, naquilo que ela incorpora as normas socioculturais que a regem, mas também naquilo que ela própria participa na reprodução e/ou na subversão dessas normas

(CHAPUIS, 2010, p. 4).

Minelle Mahtani (2004), em sua releitura geográfica do trabalho de Butler, salienta, primeiramente, como sua teorização do gênero remodelou a forma como a geografia analisa a relação entre os corpos, a produção de identidades e sua relações com o espaço. Ao mesmo tempo, ela ressalta como sua noção de performatividade contribuiu para repensar o conceito de espaço. Para explicar isso, Amandine Chapuis (2010), em seu artigo sobre a situação atual do uso da performance em geografia, cita a posição de Nigel Thrift (2000):

A abordagem não representacional, defendida especialmente por Nigel Thrift, opõe de algum modo performance e representação, sendo a representação uma imagem, uma descrição do mundo, tendo uma dimensão essencialmente intelectual, enquanto que a performance designa um corpo em ação, que produz perpetualmente uma nova realidade (CHAPUIS, 2010, p. 46).

Os trabalhos geográficos interessados na performance colocam as práticas no centro da reflexão geográfica (THRIFT; DEWSBURY, 2000). Em particular, desde a metade dos anos 1990, a geografia utiliza o trabalho de Butler para explorar a relação entre uso do corpo, produção do espaço e reprodução das normas de gênero e sexualidade ${ }^{14}$.

\section{Perspectivas para as Geografias Queers}

O referencial aqui delineado, ainda que de forma sintética, permite-nos identificar uma série de vantagens derivadas da aplicação do conceito de performance em geografia.

Primeiramente, refletir sobre a performance nos permite concentrar a atenção sobre a relação entre corpo e espaço. Isso nos permite colocar em evidência a materialidade dessa relação e as consequências sobre as transformações do espaço. Isso se mostra particularmente interessante não somente na geografia do gênero e da sexualidade, mas, também nas pesquisas sobre os movimentos sociais e sobre o uso do espaço (sobretudo urbano) no militantismo.

De fato, por meio do conceito de performance podemos revelar o caráter normalizado, heteronormalizado, regulamentado do espaço público, pois a citação das normas e sua repetição aparecem de maneira evidente quando dirigimos a atenção para os corpos e para os comportamentos dos indivíduos no espaço público. Podemos ver como o corpo 'correto' que ocupa com pleno direito o espaço público é o corpo do homem branco, ocidental, jovem e saudável: todos os que estão fora desses parâmetros são imediatamente classificados na anormalidade. Esse processo se reflete na organização dos espaços públicos, em particular urbanos, que se tornam o espaço da 'normalidade', apoiando-se numa suposta normalidade. É desse modo que um espaço considerado neutro pode tornar-se extremamente violento, uma vez que exclui os sujeitos "a-normais". No entanto, esses sujeitos possuem um potencial de subversão que pode permitir também transgredir as normas que regem os espaços públicos. As performances realizadas por certos/as atores/atrizes LGBTIQ, por exemplo, utilizam o corpo como uma ferramenta para 
reagir a um espaço público normativo e heteronormativo.

A performance faz parte de novas modalidades de militância (ION; FRANQUIADAKIS; VIOT, 2005) que caracteriza os movimentos coletivos a partir dos anos 2000. Trata-se de encenações, de ações, de representações que colocam no centro o corpo, especialmente como ferramenta de resistência às normas de gênero e como meio para tornar visível e para desnaturalizar o espaço heteronormativo.

Por conseguinte, é possível jogar com as identidades fixas para as transgredir através de suas encenações. Através da ironia e do desprezo, assistimos a uma inversão simbólica do corpo social que resiste à vontade de definição normativa. A performance exerce uma função subversiva da ordem estabelecida que oprime vozes e identidades 'outras' e permite jogar com os códigos e símbolos da heterossexualidade.

É, portanto, necessário desenvolver as pesquisas sobre o fenômeno de afirmação da identidade LGBTIQ através do uso do espaço público para compreender essa forma de expressão. Além disso, o crescimento dos episódios de agressão homofóbica em muitos países torna urgente uma reflexão sobre esse tema. A naturalização do espaço público como espaço heterossexual corre o risco, de fato, de legitimar a invisibilização dos sujeitos não-heteronormalizados e, em casos extremos, de incitar a transformação da agressão verbal e psicológica em agressão física.

A geografia das sexualidades, definida e legitimada como um ramo da geografia, pode contribuir de maneira importante para $o$ desenvolvimento das normas e das estruturas do poder que oprimem e excluem do espaço (público) os/as dissidentes sexuais. Ao mesmo tempo, como Bell e Valentine (1995a) e Valentine (1993) mostraram, ela pode questionar o caráter heteronormativo da disciplina geográfica e da instituição universitária. O papel da geografia nesse processo de desconstrução e desvelamento se mostra essencial, especialmente porque "o geógrafo pertence à categoria de 'experts' investidos de certa autoridade, ele não pode ignorar o fato de que contribui para fazer existir o que descreve" (HANCOCK, 2004, p. 172).

1 Originalmente 'L'espace à l'époque du queer: contaminations queer dans la géographie française, Revue PolitiQueer. Réflexions féministes queers francofones. p. 14-26, juin. 2014. $<$ http://politiqueer.info $>$. Tradução: Maria Helena Lenzi. Revisão: Fernando Coelho.

2 Em seu artigo ela faz uma referência explícita à geografia e ao meio acadêmico francês.

3 Para uma definiçao mais desenvolvida ver Paul Villeneuve (2003), Louargant (2002) e Borghi e Dell'Agnese (2009).

4 Para o estado da arte da relação entre Feminist studies e geografia, ver Chivallon (2001).

5 Ver, por exemplo, a questão da linguagem situada que aparecia como neutra na produção geográfica e, sobretudo, nas histórias dos exploradores (ex. "Penetrar na mata virgem") (PRATT, 1995; JACOBS, 1996).

6 Sobre a virada cultural e o 'método pósmoderno' ver Staszak (2001).

7 Na riquíssima literatura sobre esse tema, ver, por exemplo, Duncan (1996) e Barthe-Deloizy (2003).

8 Para uma revisão da literatura, ver Blidon (2008a; 2008b), Jaurand (2010; 2011) e Duplan (2012).

9 Ver o debate crítico sobre essa questão relatado por Marianne Blidon (2008b).

10 Para a geografia anglófona, ver por exemplo Valentine (1993), Bell et al. (1994), Bell e Valentine 
(1995a), Duncan (1996), Jackson (2006); para a francesa, Hancock (2004 ; 2011), Grésillon (2000), Leroy (2005; 2009; 2010), Jeraund e Leroy (2008; 2011), Jeraund (2011), Blidon (2008c; 2009), Cattan e Clerval (2011), Raibaud (2007), Sechet (2009); para a geografia italiana, Borghi e Rondinone (2009), Borghi e Schmidt (2011), Borghi (2014), Borghi e Despuches (2012). Mais raras são as pesquisas que exploram o espaço rural, como Bell e Valentine (1995b).

11 Lésbicas, gays, bissexuais, transexuais, intersexuais, queers.

12 Na realidade, em uma entrevista de 1993, Butler declara não ter conhecimento da existência de uma teoria queer da qual seria uma das principais contribuintes: "Eu sabia que Teresa de Lauretis tinha publicado um número de Differences chamado 'Queer theory'. Eu pensava que se tratava de algo que ela havia inventado. Com certeza eu não tinha alguma ideia de fazer parte da teoria queer".

13 Veremos na sequência o uso da performance para inverter a norma e não mais para reproduzi-la.

14 Para uma revisão completa da literatura, ver Mahtani (2004).

\section{Referencias}

ARFINI, Elisa. Doing gender, ovvero: che ne facciamo del genere? In: BORGHI, Liana; MANIERI, Francesca; PIRRI, Ambra (Orgs.). Le cinque giornate lesbiche in teoria. Rome: Ediesse, 2011, p. 33 - 56.

AUSTIN, John Langshaw. How to do Things with Words. Oxford: Oxford University Press, 1982.

BARTHE, Francine; HANCOCK, Claire. Introduction: le genre, constructions spatiales et culturelles. Géographie et Culture, n. 54, p. 3-10, 2005.

BARTHE-DELOIZY, Francine. Géographie de la nudité. Être nu quelque part. Paris: Bréal, 2003.
BELL, David. (et al). All hyped up and no place to go. Gender, Place and Culture, v. 1, n. 1, p. 31 - 47, 1994.

BELL, David; VALENTINE, Gill. (Org.). Mapping Desire. Londres: Routledge, 1995a.

BELL, David; VALENTINE, Gill. Queer country: Rural lesbian and gay lives. Journal of Rural Studies, v. 11, n. 2, p. 113-122, $1995 b$.

BLIDON, Marianne. Jalons pour une géographie des homosexualités. Espace géographique, v. 37, n. 2, p. 175-189, 2008a.

BLIDON, Marianne. Géographie de la sexualité ou sexualité du géographe? Remarques sur le sexe de l'enquêteur. In: COLLOQUE A TRAVERS L'ESPACE DE LA MÉTHODE: LES DIMENSIONS DU TERRAIN EN GÉOGRAPHIE, Arras, 2008b.

BLIDON, Marianne. La casuistique du baiser. L'espace public, un espace hétéronormatif. EchoGéo, n. 5, juin/août. 2008c.

BLIDON, Marianne. Genre et sexualité: deux impensés de la géographie politique française. In: ROSIÈRE, Stéphane. et al. Penser l'espace politique. Paris: Ellipses. 2009a. p. 54-68.

BLIDON, Marianne. La Gay Pride entre subversion et banalisation. Espace Populations Sociétés, n. 2, p. 305-318, 2009b.

BLUNT, Alison; WILLS, Jane. (Org.). Dissident Geographies. Harlow: Prentice 
Hall, 2000.

BONDI, Linda; DOMOSH, Mona. Autres figures en d'autres lieux: féminisme, postmodernisme et géographie. In: STASZAK, Jean-François et al. (Org.). Géographies anglo-saxones. Tendances contemporaines. Paris: Belin, 2001, p. 63 79 .

BORGHI, Rachele. Post Porn. Or, Alice's Adventures in Sexland. In: BIASIN, Enrico et al. (Org.). Porn After Porn: Contemporary Alternative Pornographies. Tourin: Mimesis International, 2014. p. 165188.

BORGHI, Rachele; DELL'AGNESE, Elena. Genere. In: DELL'AGNESE, E. (Org.). Geografia. Strumenti e parole. Milano: Unicopli, 2009.

BORGHI, Rachele; DESPUCHES, Giulia. La città velata. In: CANCELLIERI, A. et al (Org.). Tracce urbane. Milano: Franco Angeli, 2012, p. 87 - 93.

BORGHI, Rachele; RONDINONE, Antonella. (Org.). Geografia di genere. Milano: Unicopli, 2009.

BORGHI, Rachele; SCHMIDT, Marcela. (Org.). Lo spazio della differenza. Bollettino della Società Geografica Italiana, n. 1, 2011.

BUTLER, Judith. Gender as performance: an interview with Judith Butler. Radical Philosophy, n. 67, 1993.

BUTLER, Judith. Bodies That Matter: On the Discursive Limits of Sex. London/NYC: Routledge, 1993.

BUTLER, Judith. Le Pouvoir des mots.
Politique du performatif. Paris: Amsterdam, 2004

BUTLER, Judith. Trouble dans le genre. Paris: La Découverte, 2006.

BUTLER, Judith. Le pouvoir des mots: discours de haine et politique du performatif. Paris: Amsterdam, 2008.

BUTLER, Judith. Ces corps qui comptent: de la matérialité et des limites discursives du sexe. Paris: Amsterdam, 2009.

CASEY, Mark. De-dyking Queer Space(s): Heterosexual Female Visibility in Gay and Lesbian Spaces. Sexualities, n. 7, p. 446 461, 2004.

CASTELLS, Manuel; MURPHY, Karen. Cultural identity and urban structure: the spatial organization of San Francisco's gay community. In: FAINSTEN, Norman; FAINSTEN, Susan (Org.). Urban Policy under Capitalism. Beverly Hills: Sage, 1982, p. 237 - 259.

CATTAN, Nadine; CLERVAL, Anne. Un droit à la ville? Réseaux virtuels et centralités éphémères des lesbiennes à Paris. Justice spatiale, n. 3, p. 1 - 19, 2011.

CHAPUIS, Amandine. Performances touristiques. D'une métaphore à un cadre de pensée géographique renouvelé. Mondes du Tourisme, n. 2, p. 44 - 56, 2010.

CHIVALLON, Christine. Les géographies féministes. Un plaidoyer convaincant pour la constitution de connaissances 'situées'. In: STASZAK, Jean-Franpois (et al) (Org.) Géographies anglo-saxonnes. Tendences contemporaines. Paris: Belin, 2001, p. 57 62. 
CORTESI, G. Donne, società, territorio: il quadro generale. In: LOMBARDI, Daniela. (Org.). Percorsi di geografia sociale. Bologne: Patron, 2006.

COUTRAS, Jacqueline. Crise urbaine et espaces sexués. Paris: Colin, 1996.

DE LAURETIS, Teresa. Théorie queer et cultures populaire. Paris: La dispute. 2007.

DIMEN, Muriel; GOLDNER, Virginia. Gender in psychoanalytic space: between clinic and culture. New York: Other Press, 2002.

DIXON, Deborah; JONES III, John Paul. Feminist Geographies of Difference, Relation and Construction. In: AITKEN, Stuart; VALENTINE, Gill. (Org.). Approches to Human Geography. Londres: Sage, 2006, p. $42-56$.

DUNCAN, Nanci. (Org.). Body/Space. Destabilising Geographies of Gender and Sexuality. Londre: Routledge, 1996.

DUPLAN, Karine. Les géographies des sexualités et la géographie française peuventelles faire bon ménage? Géographie et cultures, n. 83. p. 117 - 138, 2012.

GREGSON, Nicky; ROSE, Gillian. Taking Butler elsewhere: performativities, spatialities and subjectivities.

Environnement and Planning D: Society and Spaces, n. 18, p. 433 - 452, 2000.

GRÉSILLON, Boris. Faces cachée de l'urbain' ou éléments d'une nouvelle centralité? Les lieux de la culture homosexuelle à Berlin. Espace géographique, n. 4, p. 301-313, 2000.

HANCOCK, Claire. L'idéologie du territoire en géographie: incursions féminines dans une discipline masculiniste. In: BARD, Christine. (Org.) Le genre des territoires: féminin, masculin, neutre. Angers: PUA, 2004, p. $165-174$.

HANCOCK, Claire. Genre, identités sexuelles et justice spatiale. Justice spatiale, n. 2 , p. $5-16,2011$.

ION, Jacques; FRANQUIADAKIS, Spyros; VIOT, Pascal. Militer aujourd'hui. Paris: Autrement, 2005.

JACKSON, Stevi. Interchanges: Gender, sexuality and heterosexuality: The complexity (and limits) of heteronormativity. Feminist Theory, v. 7, n. 1, p. $105-121$, 2006

JACOBS, Jane Margaret. Edge of empire: Postcolonialism and the City. Londre: Routledge, 1996.

JAURAND, Emmanuel. Construire des territoires d'un autre genre? Dossier HDR, (mimeo), 2010.

JAURAND, Emmanuel. Territorialités gays. ESO. Travaux et documents. n. 32, p. 7-13, 2011.

JAURAND, Emmanuel; LEROY, Stéphane. A la recherche du paradis perdu. Pratiques et représentation du littoral des touristes gays. HAL, 2008.

JAURAND, Emmanuel; LEROY, Stéphane. Tourisme sexuel: 'clone maudit du tourisme' ou pléonasme? De la sexualité dans le tourisme en général et dans le tourisme gay en particulier. Mondes du tourisme, n. 3, p. $53-65,2011$.

JOHNSTON, Linda; LONGHURST, Robin. 
Space, Place, and Sex: Geographies of Sexualities. Lanham MD: Rowman \& Littlefield, 2010.

LEROY, Stéphane. Le Paris gay. Eléments pour une géographie de l'homosexualité. Annales de Géographie, v. 114, n. 646 p. 579 - 601, 2005.

LEROY, Stéphane. La possibilité d'une ville. Comprendre les spatialités homosexuelles en milieu urbain. Espaces et Sociétés, n. 139, p. 179 - 203, 2009.

LEROY, Stéphane. La gay pride pour s'approprier l'espace public et contester la norme hétérosexuelle. HAL, 2010.

LOUARGANT, S. De la géographie féministe à la "gender geographie": une lecture francophone d'un concept anglophone. Espace Population Société, n. 3, p. 397-410, 2002.

MAHTANI, Minelle. Judith Butler. In: HUBBARD, Phill; KITCHIN, Rob. (Org.). Key Thinkers on Space and Place. Sage, 2004, p. 82 - 90.

MCDOWELL, Linda; SHARP, Joanne. A feminist glossary of Human Geography. Londre: Arnold, 1999.

NASH, Catarine. Performativity in practice: some recent work in cultural geography. Progress in Human Geography, v. 24, n. 4, p. $653-664,2000$.

PASQUINO, Monica. Se la filosofia del linguaggio incontra la politica queer. Bollettino della Società Geografica Italiana, n. 1, p. 23 - 30, 2011.

PASQUINO, Monica.; PLASTINA, Sandra. Fare e disfare. Otto saggi a partire da
Judith Butler. Milan: Mimesis, 2009.

PRATT, Mary Louise. Imperial Eyes: Travel Writing and Transculturation. Londre: Routledge, 1995.

RAIBAUD, Yves. Le genre et le sexe comme objets géographiques. In: COLLOQUE SEXE DE L'ESPACE, SEXE DANS L'ESPACE, 2007, Bordeaux. Acte du colloque de Doc' Géo, Pessac Cedex: Ades, 2007. p. 97 - 105.

RICH, Adrianne. Compulsory heterosexuality and Lesbian existance. Signs: Journal of Women in Culture and Society, v. 5, n. 4, p. $631-660,1980$.

ROSE, Gillian. Feminism and Geography: The Limits of Geographical Knowledge. Minneapolis: University of Minnesota Press, 1993.

SÉCHET, Raymonde. La prostitution, enjeu de géographie morale dans la ville entrepreneuriale. Lectures par les géographes anglophones. L'Espace géographique, v. 1, n. 38, p. $59-72,2009$.

STASZAK, Jean-Franpois. Les enjeux de la géographie anglo-saxonne. In: STASZAK, Jean-Franpois. (et al) (Org.). Géographies anglo-saxonnes. contemporaines. Paris: Belin, 2001.

Tendences

THRIFT, Nigel. Afterwords. Environment and Planning D: Society and Space, v. 18, n. 2, p. $213-255,2000$.

THRIFT, Nigel; DEWSBURY, John-David. Dead geographies - and how to make them live. Environment and Planning D: Society and Space, v. 18, n. 4, p. 411-432, 2000.

TURNER, Victor. From Ritual to Theatre: 
The Human Seriousness of Play. New York: PAJ Publications, 1982.

TURNER, Victor. The Anthropology of Performance. New York: PAJ Publications, 1987.

VALENTINE, Gill. (Hetero)sexing space: lesbian perceptions and experiences of everyday spaces. Environment and Planning D: Society and Space, v. 11, n. 4, p. 395-413, 1993.

VILLENEUVE, P. Genres. In: LÉVY, Jacques; LUSSAULT, Michel. (Org.). Dictionnaire de la géographie et de l'espace des sociétés. Paris: Belin, 2003.

WEIGHTMAN, Barbara. Commentary: Towards A Geography of the Gay Community. Journal of Cultural Geography, v. 1, n. 2, p. 106 - 112, 1981.

WIEGMAN, R., Interchanges: Heteronormativity and the desire for gender. Feminist Theory. v. 7, n. 1, p. $89-103$, 2006.

WOMEN AND GEOGRAPHY STUDY GROUP - WGSG. Geography and Gender. Random House of Canada Ltd, 1984 .. 